\title{
Co-ordinated VSOP and Chandra Observations of $0836+710$
}

\author{
D. W. Murphy
}

JPL, MS 238-332, 4800 Oak Grove Drive, Pasadena, CA 91101, USA

R. A. Preston, G. B. Piner, M. L. Lister

JPL, MS 238-332, 4800 Oak Grove Drive, Pasadena, CA 91101, USA

H. L. Marshall

Center for Space Research, MIT, Cambridge, MA 02139, USA

P. G. Edwards

ISAS, Yoshinodai 3-1-1, Sagamihara, Kanagawa 229-8510, Japan

\begin{abstract}
We briefly describe the radio observations that form part of co-ordinated Chandra, HALCA+VLBA, and VLBA-only observations of the superluminal gamma-ray loud quasar $0836+710$. The radio observations were at 6 frequencies $(1.6,5,8,15,22$, and $43 \mathrm{GHz})$ with the two lowest frequency observations being undertaken with the VLBA co-observing with the HALCA spacecraft (the space element of the Japanese VSOP mission). Combining the radio and X-ray data will allow constraints to be placed on the jet Doppler factor.
\end{abstract}

\section{VSOP Observations}

The Chandra X-ray mission observed $0836+710(\mathrm{z}=2.17)$ on October 16,2000 . As a part of a campaign to observe this source in the radio at the same epoch we observed this source on October 7, 2000 with the VLBA and the HALCA spacecraft in a special HALCA observing mode in which one $16-\mathrm{MHz}$ channel observes at $1.6 \mathrm{GHz}$ and the other at $5 \mathrm{GHz}$ while the VLBA switched between observations at 1.6 and $5 \mathrm{GHz}$ on a few minutes timescale. After our VSOP observations we further observed the source with the VLBA at 4 higher frequencies. Figure 1 shows a montage of the $1.6 \mathrm{GHz}$ and $5 \mathrm{GHz}(u, v)$-coverages along with the associated images. The tapered images are essentially the same as those made with just the VLBA while the un-tapered images show the full resolution obtainable with VSOP. By modelfitting the images with 4 distinct components we are able to determine that only the core and the closest component to the core have observed brightness temperatures which exceed the $10^{12} \mathrm{~K}$ Inverse Compton limit and consequently are expected to make significant contributions to the source's X-ray emission. By combining our radio data with the X-ray data we hope to place significant constraints on the source's jet Doppler factor. More details about our radio observations can be found in Murphy et al. 2000. 

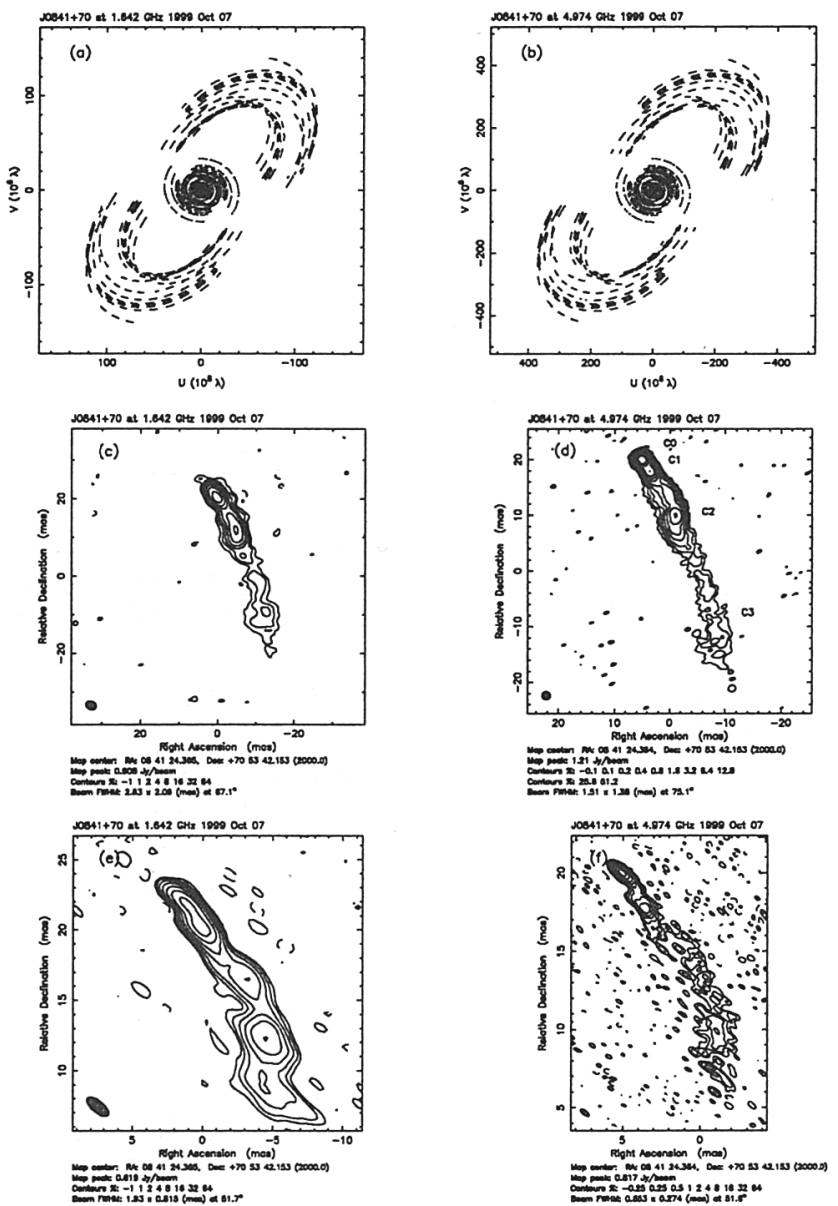

Figure 1. $1.6 \mathrm{GHz}$ and $5 \mathrm{GHz}$ HALCA+VLBA observations:

(a) $1.6 \mathrm{GHz}(u, v)$-coverage (b) $5 \mathrm{GHz}(u, v)$-coverage

(c) $1.6 \mathrm{GHz}$ image $(50 \mathrm{M} \lambda$ taper) (d) $5 \mathrm{GHz}$ image (100 $\mathrm{M} \lambda$ taper)

(e) $1.6 \mathrm{GHz}$ image (no taper) (f) $5 \mathrm{GHz}$ image (no taper).

\section{Acknowledgements}

Part of this research was performed at the Jet Propulsion Laboratory, California Institute of Technology, under contract to NASA. We gratefully acknowledge the VSOP Project, which is led by ISAS in cooperation with many organizations and radio telescopes around the world.

\section{References}

Murphy, D. W. et al. 2000, in Astrophysical Phenomena Revealed by Space VLBI, ed. H. Hirabayashi, P. G. Edwards \& D. W. Murphy, (Sagamihara: ISAS) 219 